\title{
Recalling The Heritage Of Ancient Wreath Designs: An Exploration Of The Computation In Making
}

\author{
- Sibel Yasemin Özgan \\ Istanbul Technical University, Department of \\ Interior Architecture, Istanbul, Turkey \\ sibel.ozgan@itu.edu.tr \\ - Deniz Pastutmaz Sevmen \\ Selçuk University, Department of Archeology, \\ Konya, Turkey \\ denizpastutmaz@yahoo.com
}

\begin{abstract}
The making of ancient gold wreaths is one of the unique and forgotten crafting activities, which has influenced various modern designs such as jewelries, furniture, wall decorations and ornaments. Wreath designs stand as good examples for hands-on material computation, since they are made from gold, which is known for its extraordinary durability and malleability. In this study, we try to decompose the overall rules by investigating the design development of ancient gold wreaths. The crafting activity stand as an essential attribute, which alters visual grammar rules. By decomposing the visual rules, we are able to define the relations between crafting, material properties, making techniques and visual appearance.
\end{abstract}

Keywords: Digital Heritage, Digital Craftsman, Ancient Wreath, Traditional Crafts, Computational Making

\section{Introduction}

The tendency towards crafting has existed since the beginning of will to art. It is something, which presents what a designer is actually seeing and feeling through hands-on activity.

Obviously, each making process has individual parameters; hence every single doing needs certain aptitudes. The art of making hair ornaments in ancient times is one of the unique and forgotten crafting activities, which has influenced various modern designs such as jewelries, furniture, wall decorations and ornaments. Apart from this, the continuous tradition of wearing wreaths and their later transpositions into modern forms as crowns do not only symbolize specific meanings but they exist also as an important part of practical and religious life.

Golden crowns in ancient times did not only reflect the will of a woman's grandioseness, diadems also manifested the authority and the power of politicians and emperors. Whilst their motives were used in funerary architecture and decorated building interiors and exteriors, wreaths and garlands were worn at weddings. Moreover, sculptures of ancient Greek and Roman Gods and Goddesses appear with wreaths, which are made of herbs dedicated to these Gods (Grašar \& Nikolić, 2004).

The art of making a wreath depends on the pure observation of the principles, which form the arrangement of systems in nature. Rather than simply copying the outlook of leaves to provide ornaments, these observations helped wreath makers to understand the natural operations. Thus, craftsmen were able to idealize the systems in nature to create wreaths.

As an everlasting hands-on activity, along all kinds of crafting, the making of a golden wreath has lost its reputation and significance under the imperium of mass production. Modern people do not have the time and patience to spend hours for this long-acting forming activity. Consequently, many craftsmanship has unfortunately disappeared, even as some part of the activities could be automated to manufacture identical instances of a specific design. Craftsmen and design activists such as William Morris had foreseen and pointed out the danger long before. Heritage is not only about physical artifacts, customs and traditions of a specific folk exist also as something essential to be transferred to future generations. Cultural practices and rites as wreath making signify the position of herb cult and the cyclic wreath form in a conventional sense of collective consciousness.

Whilst many crafting facilities are about to vanish, there might be a solution to the problem of maintaining this kind of intangible heritage values through computation. The maintenance of the activity might be possible in the digital era through the usage of computers. As Carpo (2011) highlights, in the early modernity, mass production through emerging cultural technologies and new machines have made identical 
replications. These copies do not inherit originality, since they all represent the same idea and principle, therefore exactly the same design. Furthermore, the automated productions of such imitations extinguish the real idea and design approach behind objects. In our days, there is a potential, which could sort out the problem. The rise of digital technologies has given an end to the power of identicality, which means eventually the end of notational limitations of industrial standardization.

This paper aims to investigate the development in ancient gold wreath designs through showing the relation between the evolutions of the elemental organizations. In this study, we try to pick up the shape rules behind the object series inside ancient elements and their making processes. During crafting activities, designers comparatively use rules and while the creative process continues, different rules emerge. The geometrical forms of ancient wreaths depend on certain symmetrical principles, which we define as the regular repetition of a shape. We investigate how each element is coming together and how this formation is ensuring the wholeness of the hair ornaments with Stiny's (2006) shape grammar formalization. While grammar formalization gives the visual rules behind the organization, material properties do explain how the forms and shapes of the each element change. Through an analysis of both material properties and visual change, this study tries to explore the ways in which the traditional processes of wreath making techniques could be incorporated with contemporary generative design processes in the realization of novel designs. Old crafts inherit precious knowledge, which could be represented, identified and enriched through computation.

The decomposition of the shape and making rules behind ancient wreaths is the first step to introduce this certain crafting technique as a possible programming language. Through the exploration of the shape rules behind ancient wreaths, we are planning to recall the crafting technique. In other words, we are aiming to encode and discover the computation in wreath making. Thus, apart from the maintenance of the activity, which may be applied to novel designs, and the transformation of knowledge in cultural heritage we are aiming to investigate the potential roles of computation in making activities. The main purpose is to create a theoretical framework for later studies by showing that the parts in the crafting activities can also be decoded. These parts are defined as the relation between material properties and crafting techniques. Thus, we seek for rules, which cannot be converted directly by visual shape grammar formalization.

In this study, we evaluate three different wreaths: two from the Hellenistic period and one from the Roman Period. We define the visual rules in order to show the relation between material properties and making.

\section{Material Properties}

The progression of jewelry designs mainly depends on the developments of tools and the experimental working on materials. Each kind of metalwork has its own properties. Experiencing different materials and trying the possibilities and limitations are what we might call material computation in modern times. Material computation appears as an essential topic in design and architecture, since it deals with the possibilities of materials, which leads to new forms. Through new fabrication techniques and robotic manufacturing, designers are now able to expand the limitations of mechanical production. Frei Otto's (1995) hands-on experimentations conducted with soap in order to explore curvilinear surfaces, which reduced eventually the surface tension is one of the best examples in material computation. In digital age, it is possible to convert the relationship between form-structure and material with computer support through computational information processing. The studies of Oxman and Rosenberg (2007), Gramazio and Kohler (2008, 2010), Menges (2010) and Oxman (2010) are relevant examples for material based computational design. In these studies, information coming from material properties, organizations and behaviors is transferred to the computer environment.

In the case of ancient wreath designs, the material used is gold, which is known for its extraordinary durability and malleability. Design decisions while exploring gold is a good example for hands-on material computation. The development of the wreath designs mainly depends on the quality of craftsmanship. In order to develop realistic acting and natural appearing leaves, a process called gold beating is done.

\section{Gold Beating}

Gold beating is the overall procedure used in order to get a thin sheet of gold, which later on was used to provide the elements of a wreath. This process is mainly divided into five sub- procedures: Melting, casting, rolling, beating and cutting and beating (Figure 1).

Although the basics are the same, gold beating in the mechanical era differs from the ancient times. Firstly, the gold and its alloys are put in a crucible and melted in a heater. Afterwards, gold in its form is poured into a mold to cast it into a bar. The bar of gold is put through a rolling mill repeatedly. Whilst the elasticity of the bars are changing, the rollers are adjusted closer and closer to each other, to make the gold thinner and thinner. The ribbon of gold is cut into small squares after rolling. The collected squares are put into a cutch, which is hammered by the craftsmen. This first hammering procedure makes thinner sheets, which later on are cut and beaten again. Thus, through the gold beating process, craftsmen obtain highly resistant and stable gold sheets. In contrast to their durability, these sheets are extraordinary thin and soft. Consequently, a soft surface of gold is obtained.

Gold beating has been used since the ancient Egyptians, who are the first practitioners of the art. The drawings on tombs at Saqqara and Thebes show their goldbeaters working 
together with gold founders and goldsmiths. Because of their appearance and stability, these sheets could be employed to gold wreaths (Nicholson, 1979). In modern era, we might describe the overall process as simple since we can use the help of machines. However, the craftsmanship in ancient times depended more on muscle force. After the gold beating process provided thin sheet, craftsman formed the elements of a gold wreath with a usage of a mold, which helped them to get repetitive shapes with minimum effort.

In this study, we try to understand the design development of ancient gold wreaths by revealing the connection of crafting procedures and materialization. In order to get more sophisticated elements, the beating process had to last for many hours.
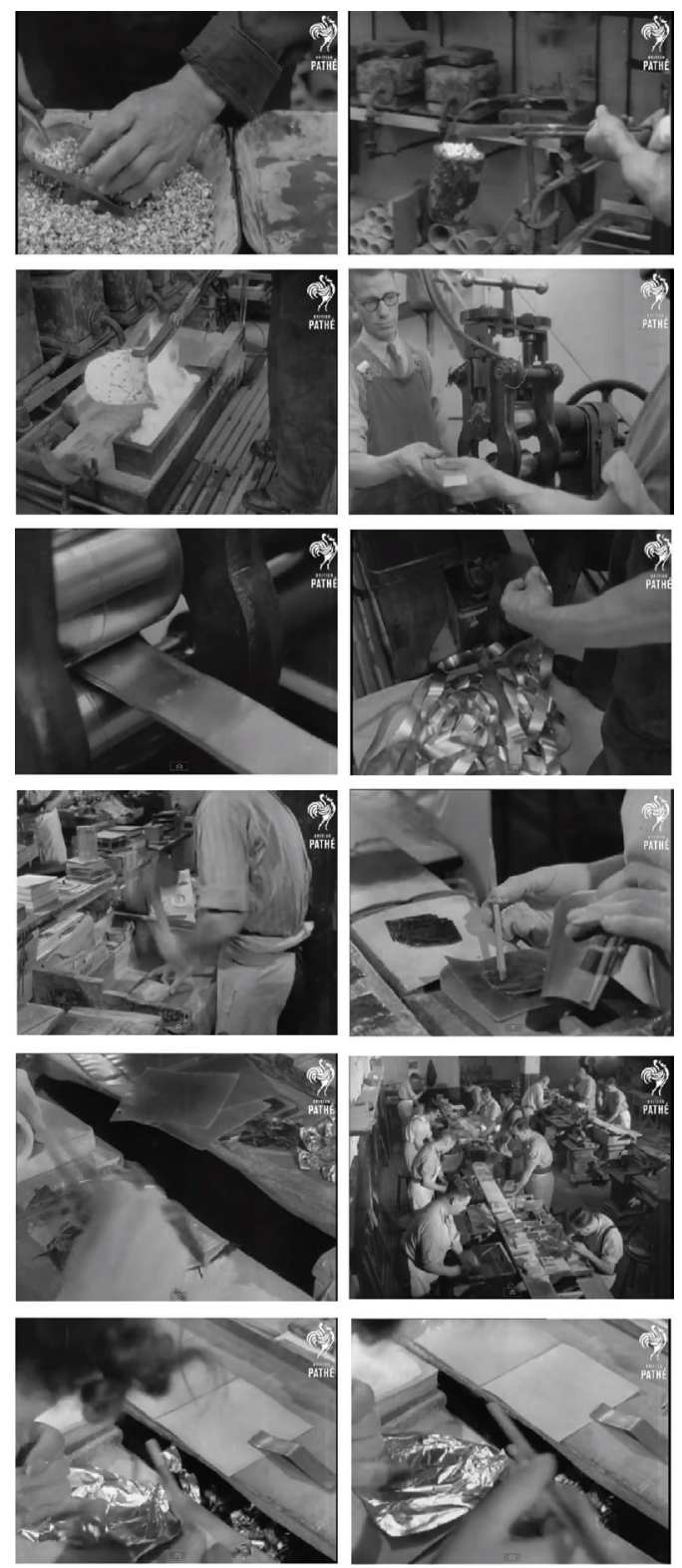

Figure 1: Gold beating process in the mechanical age. The procedures are: Melting, Casting, Rolling, Beating and Cutting and Beating again. Taken from BIF (n.d).

\section{Design Development}

In order to build a wreath, a geometric construction had to be planned by the craftsman. These geometric constructions needed strategically planning of all the elements, which were basically leaves, grasses, flowers or branches. These fundamental components differed also for each culture. As an example, according to specific meanings for each ancient civilization, leaf elements vary in forms of laurel, oaken, myrtle, olive, ivy \& grapevine wreaths and other herbs and fruits. The selection depends on how each folk symbolize these individual components.

In this section, different wreath examples from the Hellenistic and Roman Periods are investigated to expose the interaction between materials, making and visual rules. The difference in making of these wreaths is caused firstly by the beating time of the gold. The more the gold is beaten, the more flexible and realistic appearance is provided. Thus, the organization on the wreath changes, since the element weight changes accordingly.

\section{Hellenistic Period}

The 'Edinburgh Wreath' (figure) is formed through thin branches and $5 \mathrm{~cm}$ long leaves, which are attached to a main cylindirical wire body. This branch shaped main body is structured with two circular sectioned golden pipes connected to each other from back and front sides with thin golden wires.

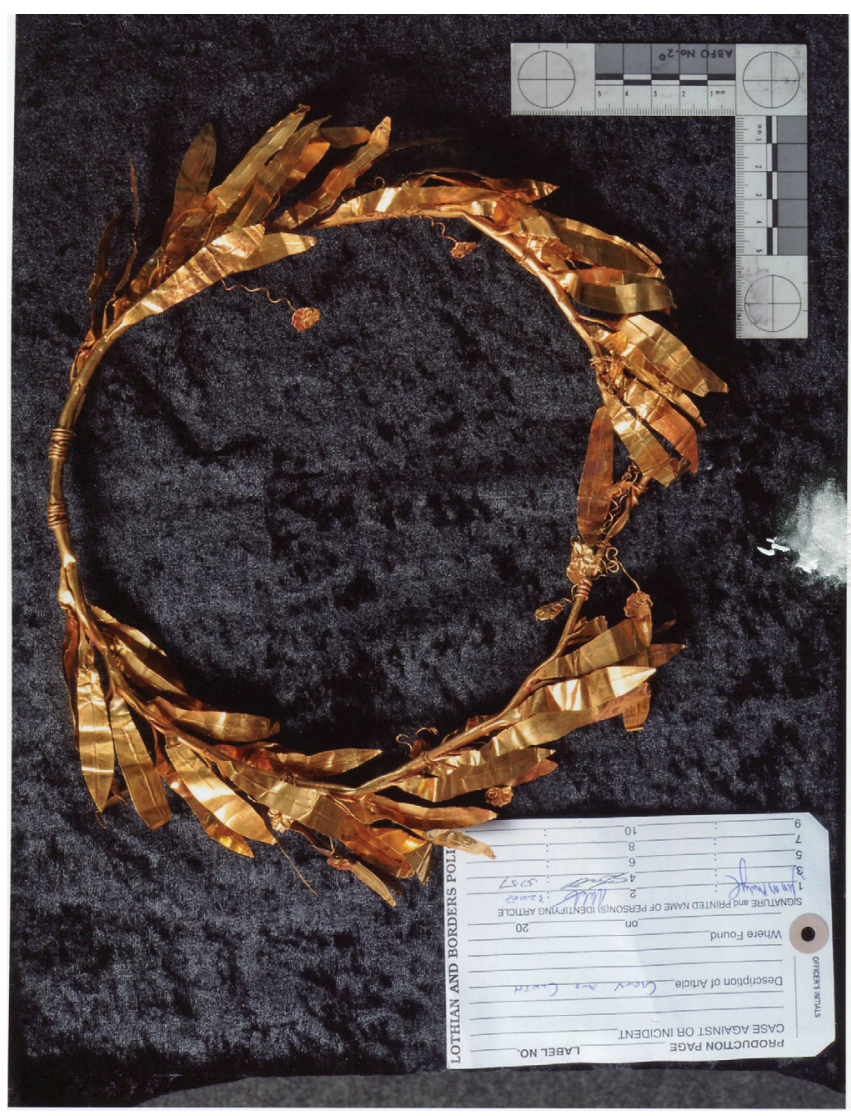

Figure 2: The Edinburgh Wreath. 
Holes are bored into the main body. The small elements of the wreath- leaves and branches- are joined to the main body from these wholes by simply twining the bottom branches. Thin strings are beaten on to the surfaces with the intention of creating a more realistic view. More than twenty leaves are placed on to each other's surfaces.

The pointed ends of the leaves in both sides are facing each other. The frequency and the workmanship of the leaves provide the impressive view, which is extended by the partly used branches with different thicknesses. The relatively thick and twisted branches located in the back of the wreath, have also a functional feature: attaching winding through these branches stabilizes the main body joint. Thus, this kind of elements is used as a link, as well as components providing aesthetics without any interruption or division. Some of the branches are made more spiral and the thinner ones have flower shaped ornamental elements in their endings. These thin branches with flower rosettes are not only located in the free parts left from the overall organization, but they are also located between the leaves. Beating the gold leaves on a flower shaped matrix forms these pin-flowers, so they stand as a whole rather than structured as attached independent leaves.

Another example is a naturalistic wreath of oakleaves and acorns from Dardanelles (Figure 3). The wreath is supported on two golden branches. In this example, we see other ornaments in fine quality. The branches finish in obliquely cut end-plates at the back, while they are arranged together by a split pin fastener concealed by a golden bee at the front. Different than the first example, the leaves and other elements are fastened on to prays. There are six sprays with eight leaves and seven or eight acorns, as well as a cicada. Additionally, about a dozen single leaves are attached directly to each branch.

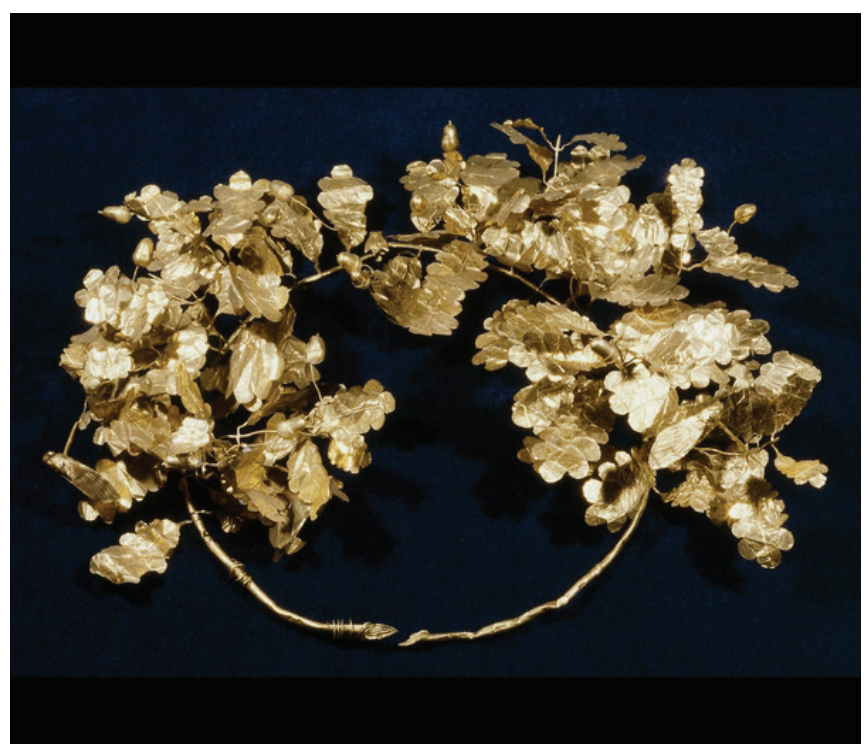

Figure 3: The Golden Wreath from Dardanelles. Taken from the official British Museum website.

\section{Roman Period}

Compared to the Hellenistic examples, Roman wreath designs are much more simple. Since the realistic look of the leaves depend on the long hammering hours of gold, Although Hellenistic examples present earlier designs, the craftsmanship in ancient Rome is not as sophisticated as in the ancient Greece. Thus, a more humble look is seen. The nature like appearance in the earlier examples vanishes in this period.

Figure 4 shows a Roman golden wreath located in Çorum Museum. In contrast to the Hellenistic examples, the main body of this wreath is a thin golden band. The leaves are directly attached to the main body by opening a hole on their ends. Wires hammered as thin bands are placed into these holes. Functioning as a kind of staple, through the usage of these thin bands the elements are attached to the main body.

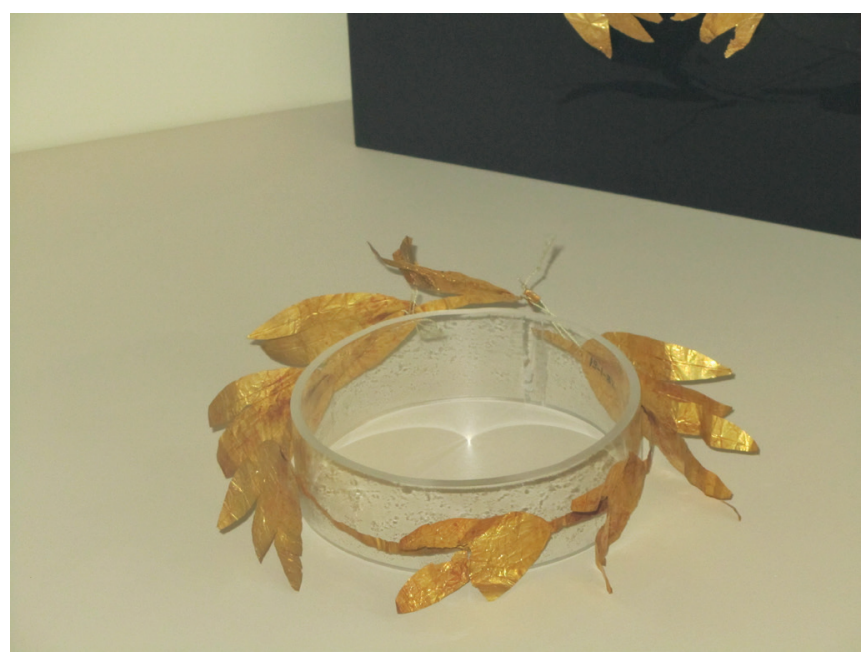

Figure 4: The Golden Wreath from Dardanelles. Taken from the official British Museum website.

The examples from the Hellenistic period (particularly from $\mathrm{BC} 4 \mathrm{BC} 3$ ) are designed with many small elements such as leaves, flowers and sometimes fruits. In contrast to the Roman examples, the elements in the Hellenistic wreaths are not directly attached to the main body. Through branches, the leaves stand not that close to the main part. Consequently, small elements look as they are flying just like real leaves flying through the wind. In Roman wreaths, the number of the elements used decreases, since the hammering hours shrink.

The main body much more simple, a thin bant, is created using a small amount of gold. The leaves are far from being natural, in the form of stylized flowers.

\section{Evaluation}

In all of the examples given above, although the leaves are produced from the same matrix, the thickness of the base material- the gold leaf- alters each shape. The naturalistic look is provided through these differentiations. Just as it is in the nature, leaves look exactly the same in the overall 
look, however through the material properties, bending and twisting degrees are altered. Therefore, it is impossible to talk only about the visual rules and simple symmetrical rules. Figure 5 shows grammar rules of the Edinburgh wreath. It is clearly seen that visual rules do not present the real-time look of the wreath. Since the material is really flexible each leave alters in their forms.

Due to their more rigid material, Roman wreaths (Figure 6), look more alike their visual rules.
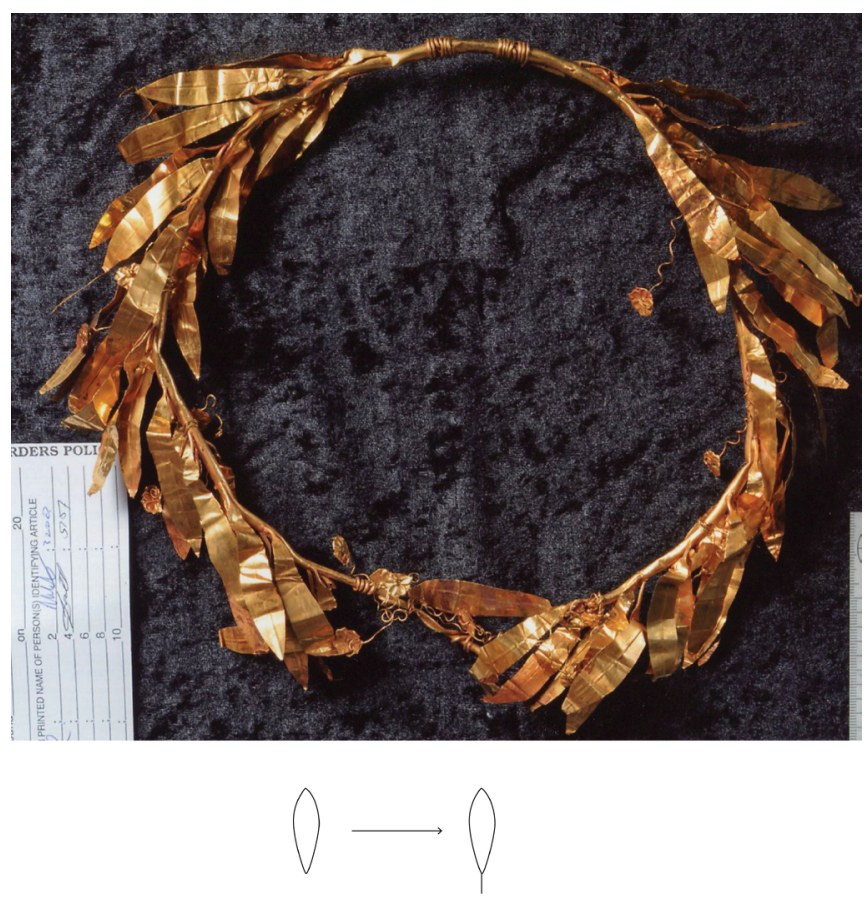

Rule 1

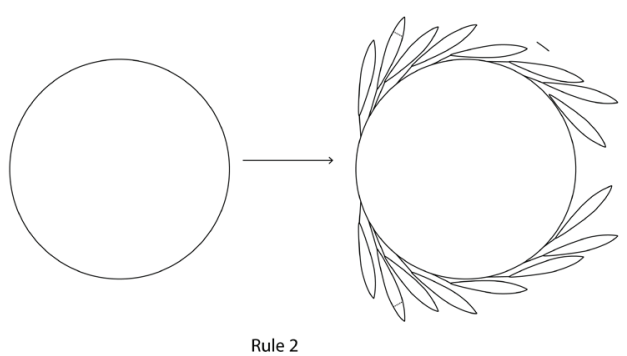

Hellenistic Wreath from Edinburg

beating time: 5 hours

thickness of the material: $0,03 \mathrm{~mm}$

type of connection to the main body: wires

Figure 5: Visual rules and extra attributes of the Edinburg wreath.

In these different examples, if we define the hammering times as extra attributes, we are able to calculate the movement of the gold material. The more the gold is beaten, the more malleable elements are obtained.
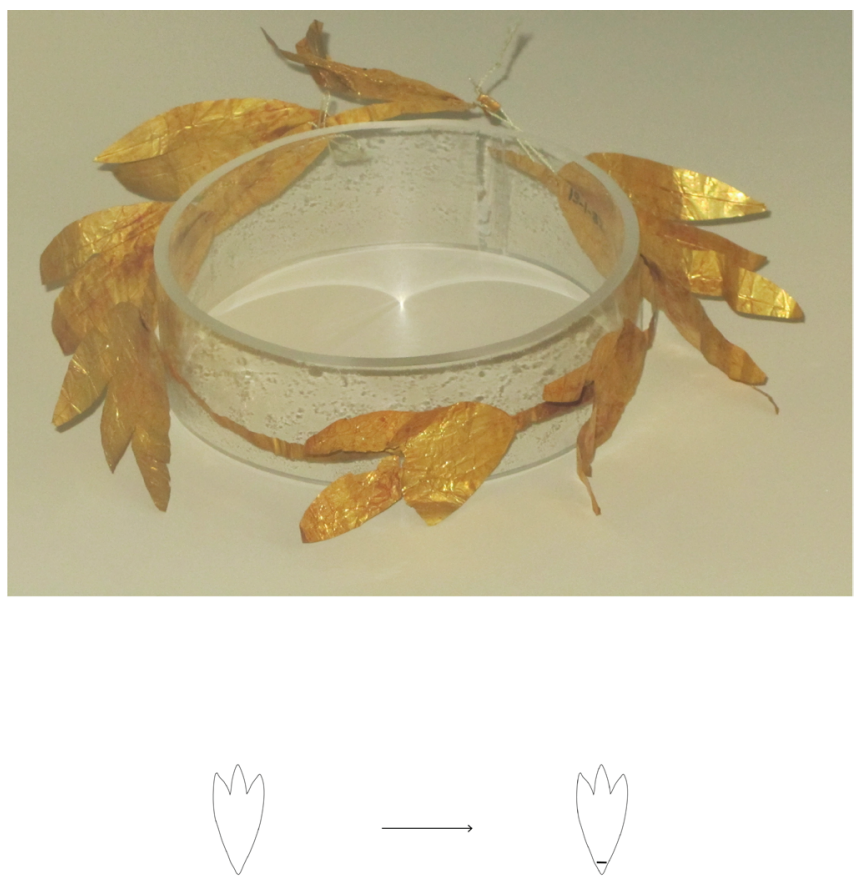

Rule 1

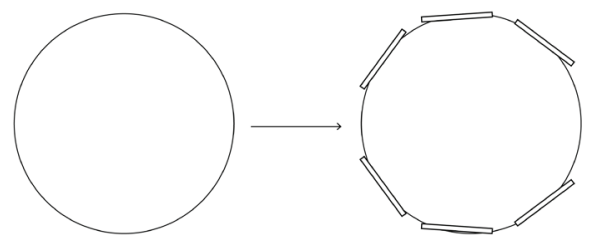

Rule 2

Roman Wreath from Çorum

beating time: 1 hour

thickness of the material: $0,05 \mathrm{~mm}$

type of connection to the main body: direct

Figure 6: Visual rules and extra attributes of the Roman wreath.

The calculation of the elements by their material properties is decoded through a grid system (Figure 6 and 7). The proportions and scaling of the system is altered according to their malleability. The grid system expands as the beating times decrease. Although all the elements are taken from the same molds, each form acts differently. In the three examples given above, the most naturalistic design is in the making of the Hellenistic wreath from Dardanelles. The squares of the grid system in this example are the smallest ones. Thus, the system explains why the elements from the same matrixes are diverse. 


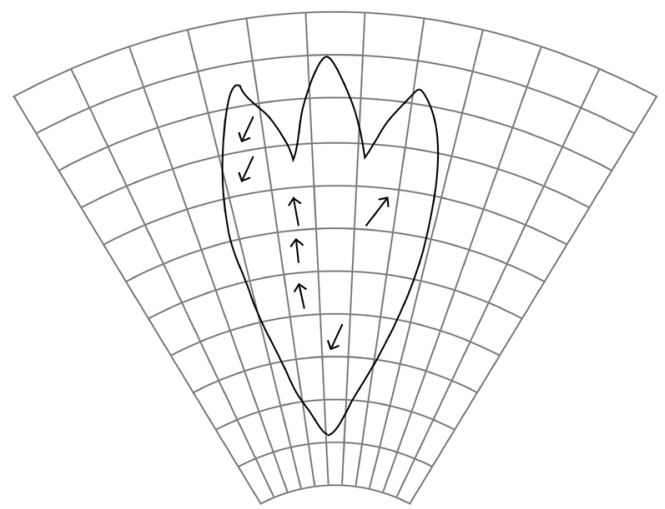

Roman Wreath from Çorum

beating time: 1 hour

thickness of the material: $0,05 \mathrm{~mm}$

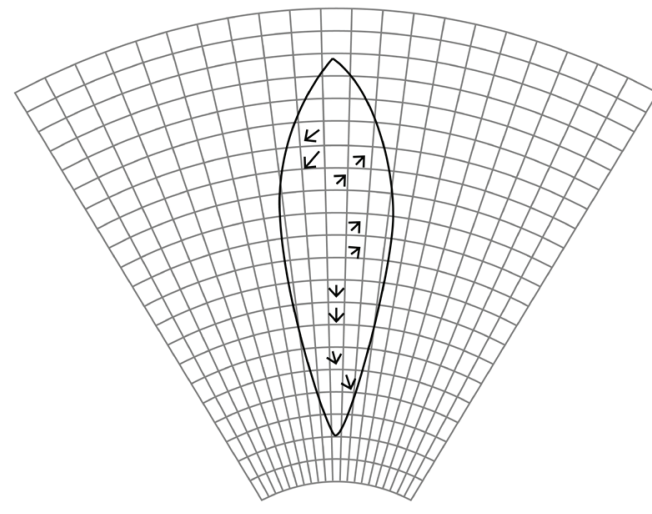

Hellenistic Wreath from Edinburg

beating time: 5 hours

thickness of the material: $0,03 \mathrm{~mm}$

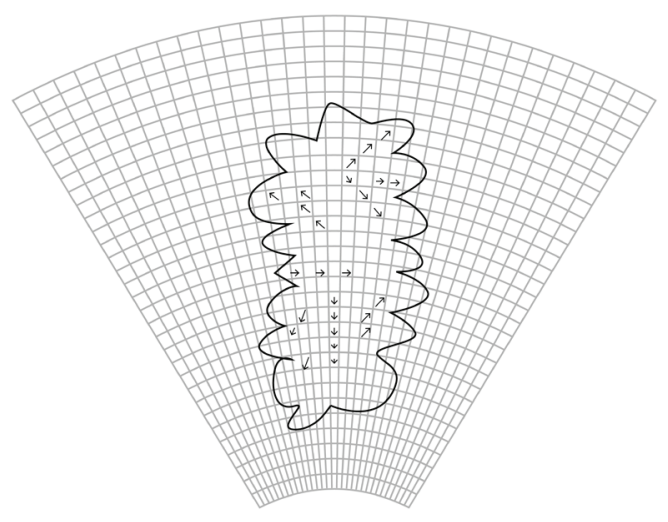

Hellenistic Wreath from Dardanelles

beating time: 10 hours

thickness of the material: $0,01 \mathrm{~mm}$

Figure 7: Grid system to calculate elemental attributes.
+ direction

bending resolution shape connection

$\mathrm{mm}$

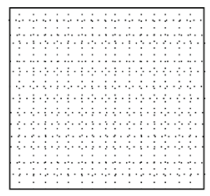

$\{\xi$

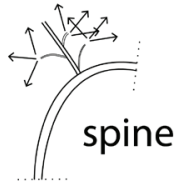

0.03

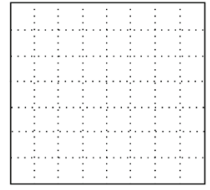

$\lambda$<smiles>C#CC#CC=C</smiles>

0.05
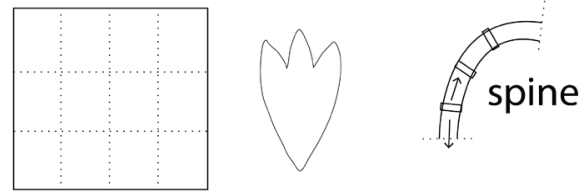

Figure 8: Parameters of different wreath designs.

\section{Conclusion}

In design activities, it is impossible to talk only about visual calculation. The ambiguity in materialization leads designers to push the limits of materials and get unexpected encountering through the process of making. We can only explain the computation in design activities by understanding various parameters of a specific action.

In this study, we try to explain the making processes of ancient wreaths through revealing the visual rules and material computation behind.

We evaluate three different examples by visual rules and other parameters such as material beating time, material thickness and connection types. Thus, we seek for rules, which cannot be converted directly by visual shape grammar formalization. We give a theoretical framework for later studies by showing that the parts in the crafting activities can also be decoded. These parts are defined as the relation between material properties and crafting techniques. This first step of the decomposition of the shape and making rules behind ancient wreaths can later be extended by introducing this certain crafting technique as a possible programming language

\section{References}

BIF, British Instructional Films, Gold Beating (n.d.), Retrieved from https://www.youtube.com/watch?v=Z4B6o2MmITw Carpo, M. (2011). The alphabet and the algorithm. MIT Press. Gramazio, F., \& Kohler, M. (2008). Digital materiality in architecture. Lars M€uller Publishers.

Gramazio, F., Kohler, M., \& Oesterle, S. (2010). Encoding 
material. In R. E. Oxman, \& R. M. Oxman (Eds.), Architectural design: The new structuralism: Design, engineering and architectural technologies (sf. 108e115). John Wiley \& Sons. Grašar, J. A., \& Nikolić, E. (2004). Wreath its use and meaning in ancient visual culture. Religion and tolerance, 341. Hunter, N. (2012). The art of floral design. Cengage Learning. Jones, O. (1868). The grammar of ornament. B. Quaritch. Iversen, M., \& Riegl, A. (1993). Alois Riegel: Art History and Theory. MIT Press.

Knight, T. W. (1995). Transformations in design: a formal approach to stylistic change and innovation in the visual arts. Cambridge University Press.

Knight, T. W. (1998). Infinite patterns and their symmetries. Leonardo, 305-312.

Knight, T., \& Stiny, G. (2001). Classical and non-classical computation. arq: architectural research quarterly, 5(04), 355372.

Nicholson, E. D. (1979). The ancient craft of gold beating. Gold Bulletin, 12(4), 161-166.

Otto, F. ve Rasch, B., 1995, Finding Form, Edition Axel Menges. Otto, F. ve Rasch, B., 1982, Natürliche Konstruktionen; DVA.

Oxman, N., \& Rosenberg, J. L. (2007). Material-based design computation: An Inquiry into digital simulation of physical material properties as design generators. International Journal of Architectural Computing, 5(1), 26-44.

Oxman, N. (2010). Material-based design computation (PhD Thesis, Massachusetts Institute of Technology).

Riegl, A. (2004). Historical grammar of the Visual Arts (p. 170). New York, NY: Zone books.

Stiny, G. (1980). Introduction to shape and shape grammars. Environment and planning B, 7(3), 343-351.

Stiny, G. (2008). Shape: talking about seeing and doing. The MIT Press.

The British Museum, Gold Wreath (n.d), Retrieved from the official museum website. http://www.britishmuseum.org/ explore/highlights/highlight_objects/gr/g/gold_wreath.aspx

Treister, M. Y. (Ed.). (2001). Hammering techniques in Greek and Roman jewellery and toreutics (Vol. 8). Brill.

Washburn, D. K., \& Crowe, D. W. (1988). Symmetries of culture: Theory and practice of plane pattern analysis. University of Washington Press.

Wade, D. (2006). Symmetry: The ordering principle. Bloomsbury Publishing USA.

Weyl, H. (1952). Symmetry. Princeton University Press. 